\title{
Application of the Fuzzy Modeling for Optimization of Transport Routes
}

\author{
I. B. Olenych ${ }^{1, *}$, A.F. Gukaliuk ${ }^{2}$ \\ ${ }^{1}$ Department of Electronics and Computer Technologies, Ivan Franko Lviv National University, Ukraine \\ ${ }^{2}$ Department of Economics, Ivan Franko Lviv National University, Ukraine
}

Copyright $\bigcirc 2017$ by authors, all rights reserved. Authors agree that this article remains permanently open access under the terms of the Creative Commons Attribution License 4.0 International License

\begin{abstract}
The expert system of the fuzzy inference of the Sugeno's type with three inputs and one output for the choice of optimal routes of the transport network is proposed in the work. The developed system involves the formation of a fuzzy production rules base, the fuzzification of the values of the input parameters, the aggregation of the truth of the sub-conditions of each rule, activating the conclusions and defuzzification the output variable. An algorithm for finding quantitative values of coefficients that corrects the weight of the edges of the graph in optimization problems under conditions of approximate input data is implemented. In particular, the proposed model takes into account the condition of the road surface, the urgency of delivery and the caution of transportation of cargoes during the formation of transport routes, which allows minimizing costs and improving the efficiency of the logistics system.
\end{abstract}

Keywords Transport Routes, Fuzzy Modeling, Production Rules, Fuzzy Sets

\section{Introduction}

The rational organization of logistics operations and functions related to the movement of products by vehicles occupies an important place in the network of business processes in conditions of deepening globalization [1]. The use of innovative technologies and new IT tools, including e-commerce, along with the development of transport infrastructure and the integration of all functions of supply management, improve the economic efficiency of the logistics chain [2-5]. Transportation is one of the key logistics functions that combine separate types of production procedures, from manufacturing to delivering products to consumers. Optimization of transport costs largely determines the efficiency of the entire logistics system [6].

Choosing the best routes and delivery schemes is an important task in providing forwarding services, which is solved through a comparative analysis of all possible options. The optimal route allows not only to guarantee the timely fulfillment of the requirements of the consumer, but also to substantially reduce the cost of delivery of the cargo, making it at the same time as fast and competitive as possible. From the socio-economic point of view, this will ensure the preservation of human and energy resources.

Effective solutions in logistics-oriented systems usually require the prompt consideration of many factors that are characterized by weak structuring, fuzzy interactions, incompleteness and approximation of the formal description, etc. Modeling is often used in research in the fields connected with production, strategic management, planning and organization of movement of material and information flows [7, 8]. For the proper design and analysis of models, the theoretical basis used for other disciplines such as economics, marketing, social sciences and engineering $[9,10]$ is applied. With transport logistics the classical optimization problems of the theory of graphs are inextricably linked - the problem of finding the shortest path (for example, the Dijkstra's algorithm and Floyd-Warshall algorithm) and the minimal spanning tree (in particular Prim's algorithm algorithms and Kruskal's algorithm) [11]. In these tasks the weight of the edges, which make up the path or the spanning tree, is minimized. It should be noted that an arbitrary transport system can be represented in the form of a weighted graph, and the weight of the edge in the graph can be not only the distance, but also the cost of transportation, time or any other criterion that is user-targeted. However, such algorithms do not take into account the additional influence of various factors that can change the weight of the edge in the graph. In addition, these factors may change over time, which requires a quick correction of the model of the transport system.

Additional complexity in the formation of routes 
predetermines the need to comply with the necessary conditions of carriage, in particular taking into account the condition of the road surface, the requirements for careful transport, the urgency of cargo delivery, etc. Comprehensive coverage of various criteria in determining the optimal routes involves the use in mathematical models of the organization of communications of linguistic variables, which, according to the presentation structure, approach to the spoken language. In this case it is advisable to use methods of fuzzy modeling. The theory of fuzzy sets makes it possible to apply a linguistic description of weakly structured processes, to establish a correlation between concepts and formalize fuzzy rules in decision making information systems [12-15].

The purpose of this work is to modify optimization network algorithms by introducing corrective factors that take into account the parameters of the transport system and the conditions of transportation. Particular attention has been paid to the formalization of qualitative and approximate parameters of the logistic system model by means of fuzzy logic. Such an approach made it possible to determine the corrective factors and to analyze the impact of the road condition, the urgency of delivery and the caution of the carriage on their quantitative values.

\section{Model Description}

The application of methods of fuzzy modeling allows you to calculate the coefficients that correct the weight of the edges of the graph in optimization algorithms, depending on the transport conditions. In this case, the minimum path $S$ will be determined not only by the weight of the $i$-edges of the graph $a_{i}$, which models the transport system, but also by the value of the corrective factor $k_{i}$, which reflects the transport conditions for each of the sections of the logistics network:

$$
S=\sum_{i} k_{i} a_{i} \rightarrow \min , \quad k_{i}>0
$$

The value of the corrective factor $k_{i}$ may be $<1$ in the case that the resulting effect of all conditions of product movement in the selected area $a_{i}$ of the network minimizes costs by a certain criterion, and vice versa $->$ 1 , if such influence reduces the efficiency of the logistic system. Thus, depending on the transportation parameters, the effective length of the transport network segment (i.e., the weight of the corresponding edge of the graph) may decrease or increase. The use of corrected data in known algorithms for finding the minimum path will allow you to choose the best route, taking into account the conditions of transportation, and respond promptly to their change.
In the general case, the corrective factor can be determined by a linear combination of quantitative values of all parameters $x_{j}$ that affect the transportation of products in the $i$-segment of the transport network:

$$
k_{i}=\sum_{j} \varepsilon_{j} x_{j}
$$

where $\varepsilon_{j}$ - weight coefficients of each of the parameters. The proposed approach makes it possible to apply Sugeno's algorithm to calculate the corrective factors, according to which a linear combination of clear values of input variables forms the conclusion of each of the fuzzy production rules $[16,17]$. The formalization of approximate considerations in such expert systems is that, based on a certain degree of truth of fuzzy statements, which are a condition of production rules, to assess the degree of truth of fuzzy statements, which are the conclusions of these rules. For this purpose it is necessary to form the basis of the rules of the system of fuzzy conclusion in the form IF (condition) - THEN (action), to fuzzificate the values of the input parameters, to carry out the procedures for activating the conclusions and defuzzification of the output variable $[17,18]$.

\section{Design of Fuzzy Inference System}

The algorithm for obtaining fuzzy logical inference was implemented for the model of the formation of transport routes, which takes into account three important parameters of transportation conditions. In particular, such parameters are the condition of the road surface, the urgency of delivery and carefulness of transportation of cargoes. In the proposed model bad road conditions and a high level of carefulness of transportation increase the corrective factor, while increasing the urgency of delivery reduces it.

In accordance with the model an expert system of the fuzzy inference of the Sugeno's type was developed with three inputs and one output. The setting of input linguistic variables "road conditions" = \{"bad", "satisfactory", "good"\}, "level of caution" = \{"low", "high"\} and "urgency" = \{"little", "great" $\}$ in plural [0, 10] allows experts to quantify the criteria of a transport system on a ten-point scale. Membership functions of fuzzy sets characterized the terms of the input variables "road conditions", "level of caution" and "urgency" were set by piecewise linear, as shown in Figure 1. With the help of the set membership functions, the quantitative values of the parameters of transportation can be fuzzificate. As a result of activating the conclusions of fuzzy production rules at the output of the expert system, one can obtain the corrective factor $k_{i}$ for each edge of the graph that characterizes the transport and logistics infrastructure. 


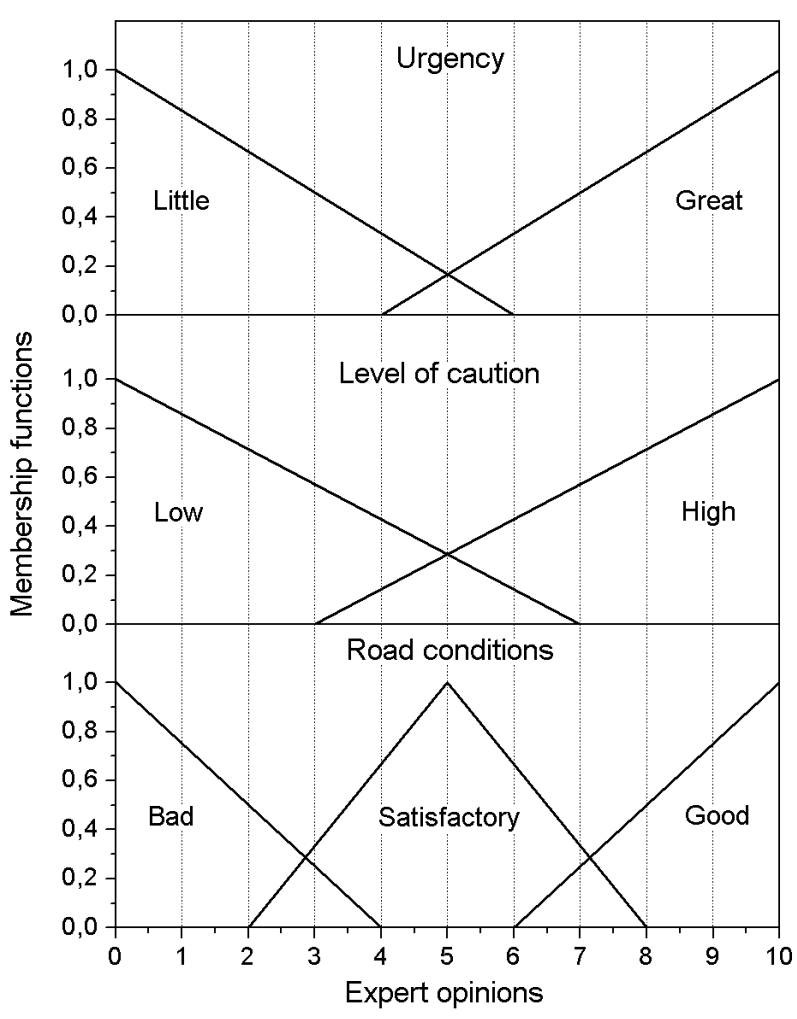

Figure 1. Membership functions of fuzzy sets characterized the terms of the input variables "road conditions", "level of caution", "urgency"

The mechanism for obtaining a fuzzy inference is based on information of various origin, in particular experimental data, simulation results and expert opinions, which are presented in the form of fuzzy production rules $R^{(j)}$ :

$\mathbf{R}^{(1)}$ : IF bad road conditions AND great urgency

THEN $w_{1}=1,5-0,05 x_{1}-0,02 x_{2}$;

$\mathbf{R}^{(2)}$ : IF satisfactory road condition AND great urgency

THEN $w_{2}=1,4-0,04 x_{1}-0,025 x_{2}$;

$\mathbf{R}^{(3)}$ : IF good road conditions AND great urgency

THEN $w_{3}=1,2-0,02 x_{1}-0,035 x_{2}$;

$\mathbf{R}^{(4)}$ : IF bad road conditions AND little urgency

THEN $w_{4}=1,3-0,025 x_{1}-0,055 x_{2}$;

$\mathbf{R}^{(5)}$ : IF satisfactory road conditions AND little urgency

THEN $w_{5}=1,2-0,02 x_{1}-0,05 x_{2}$;

$\mathbf{R}^{(6)}$ : IF good road conditions AND little urgency

THEN $w_{6}=1,1-0,01 x_{1}-0,04 x_{2}$;

$\mathbf{R}^{(7)}$ : IF bad road conditions AND high level of caution

THEN $w_{7}=1,4-0,04 x_{1}+0,03 x_{3}$;

$\mathbf{R}^{(\mathbf{8})}$ : IF satisfactory road conditions AND high level of caution

THEN $w_{8}=1,04-0,04 x_{1}+0,02 x_{3}$;

$\mathbf{R}^{(\mathbf{9})}$ : IF good road conditions AND high level of caution

THEN $w_{9}=1-0,025 x_{1}+0,01 x_{3}$;

$\mathbf{R}^{\mathbf{( 1 0 )}}$ : IF bad road conditions AND low level of caution

THEN $w_{10}=1,2-0,05 x_{1}+0,04 x_{3}$;

$\mathbf{R}^{(11)}$ : IF satisfactory road conditions AND low level of caution
THEN $w_{11}=1,1-0,03 x_{1}+0,02 x_{3}$;

$\mathbf{R}^{(\mathbf{1 2 )}}$ : IF good road conditions AND low level of caution

THEN $w_{12}=1-0,04 x_{1}+0,02 x_{3}$.

Here $x_{1}, x_{2}, x_{3}$ are a quantitative assessment of the road conditions, degree of urgency and caution, respectively.

The algorithm for obtaining corrective factors for optimizing the transport system routes contains the following stages of fuzzy simulation:

1. Using the fuzzification procedure for the set values of the input variables, the degree of truth of the statements of each of the terms of the linguistic variables that formed the condition of the production rule $R^{(j)}$ is determined. It should be noted that the degree of truth of the statement is defined as the value of the membership function of the corresponding fuzzy set for a set quantitative value of the selected parameter of the transportation.

2 . The set $C=\left\{c_{j}\right\}, j=1 \ldots n$ of the values of the truth of the rules of the system of the fuzzy conclusion are obtained by aggregation of the degree of truth of assertions, which are the subconditions in each of the fuzzy rules. The aggregation procedure is carried out by means of a fuzzy conjunction operation AND.

3. The truth degree of conclusion $w_{j}$ for each fuzzy production rule is determined by a linear combination of the quantitative values of the input parameters of the transportation. In the conclusions activation procedure, only active production rules are taken into account, the truth degree of conditions for which is different from zero.

4. Since in the Sugeno's type algorithm the conclusion of fuzzy rules is formed by a linear combination of the usual input values, therefore the value of the output variable is determined by a real number and there is no procedure for the accumulation of conclusions. The determination of the corrective factor $k_{i}$ is carried out by means of the defuzzification by the center of weight method for single-element sets [16]:

$$
k_{i}=\frac{\sum_{j=1}^{n} c_{j} w_{j}}{\sum_{j=1}^{n} c_{j}}
$$

where $n$ - number of fuzzy production rules.

The application of the proposed algorithm for each area of the network, which is characterized by its set of transportation parameters, allows determining the values of the coefficients that correct the weight of the edges of the weighted graph of the logistic network.

\section{Results and Discussion}

Figures 2-4 illustrate the results of testing the expert system for optimizing transport routes based on the fuzzy inference, where the dependences of correction factor from the considered transportation parameters expressed 
on the 10-point scale are shown.

Figure 2 shows surfaces $k=f\left(x_{1}, x_{2}\right)$ input-output of the system of the fuzzy inference with fixed values of the level of caution of transportation. The obtained results demonstrate that the good road condition and high urgency provide low values of the correction factor for the selected transport network segment. High value of the level of caution of transportation $\left(x_{3}=10\right)$ causes an increase of the correction factor. To a greater extent such growth is observed in the region of low values of the parameter $x_{1}$, which corresponds to the bad state of the road.

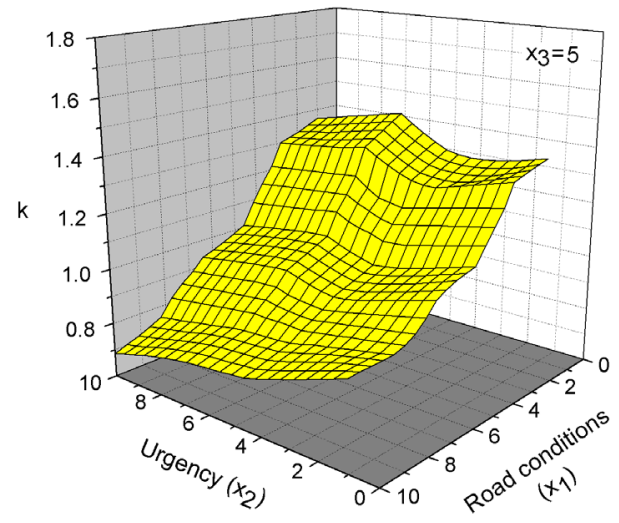

a)

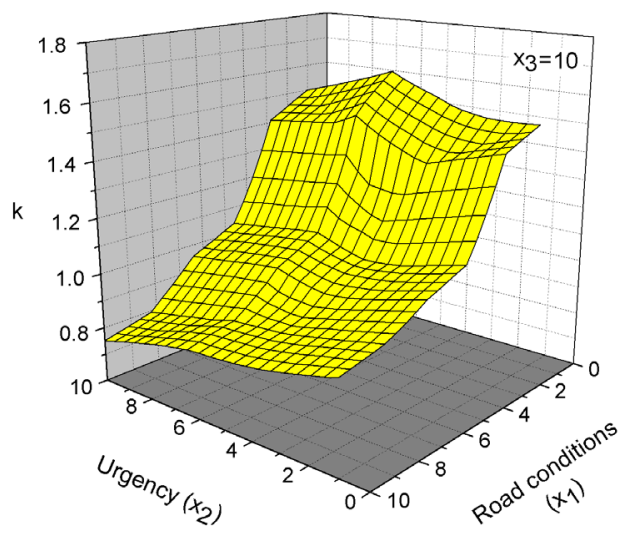

b)

Figure 2. Dependence of the correction factor on the road condition and the urgency of transportation at moderate (a) and high level of caution (b)

The dependence of the correction factor on the road condition and the level of caution of transportation $k=f\left(x_{1}\right.$, $x_{3}$ ) is shown in Figure 3. The highest values of the factor are observed for parameters that characterize the bad road condition and the high level of caution of the transportation. The urgency of transportation almost does not affect the character of the surface received by the expert system on the basis of fuzzy inference.

In the case of visualization of the obtained results in the form of a surface $k=f\left(x_{2}, x_{3}\right)$ it has been revealed the reduction of the correction factor at high urgency and the low level of caution for a good road condition $\left(x_{1}=10\right)$ and the values of the factor close to 1 for the satisfactory road condition $\left(x_{1}=5\right)$ and for arbitrary values of other parameters of the logistic network model (see Figure 4).

For better visibility the results of testing the intellectual system for determining the factors that correct the weight of the edges of the graph in optimization algorithms for the formation of transport routes for different transportation conditions are shown in Table 1.

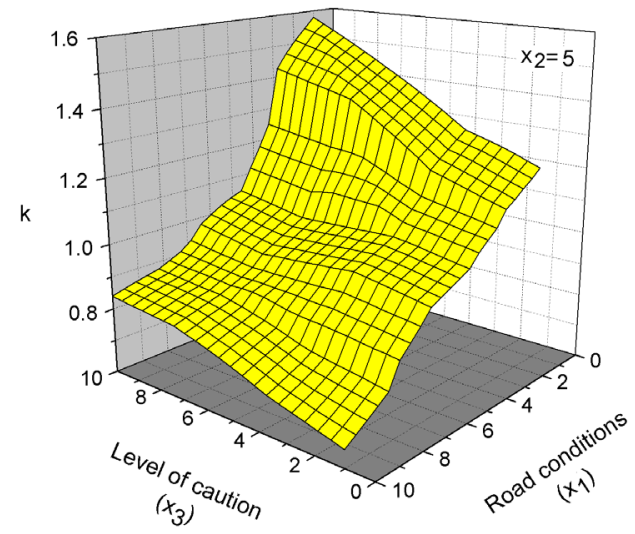

a)

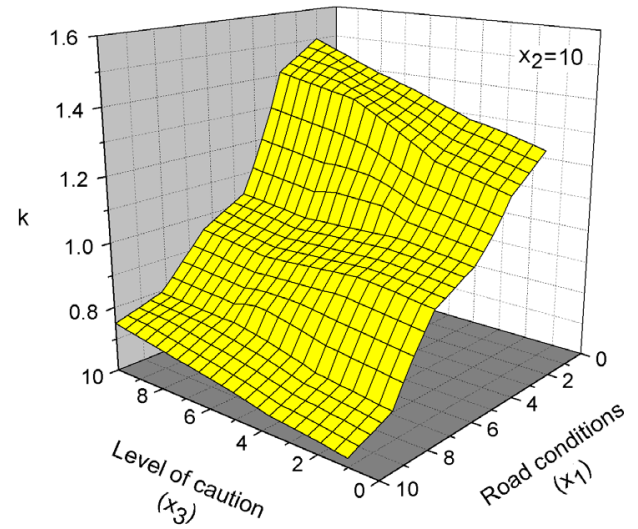

b)

Figure 3. Dependence of the correction coefficient on the state of the road and the level of care of the transport at moderate (a) and high (b) urgency of delivery

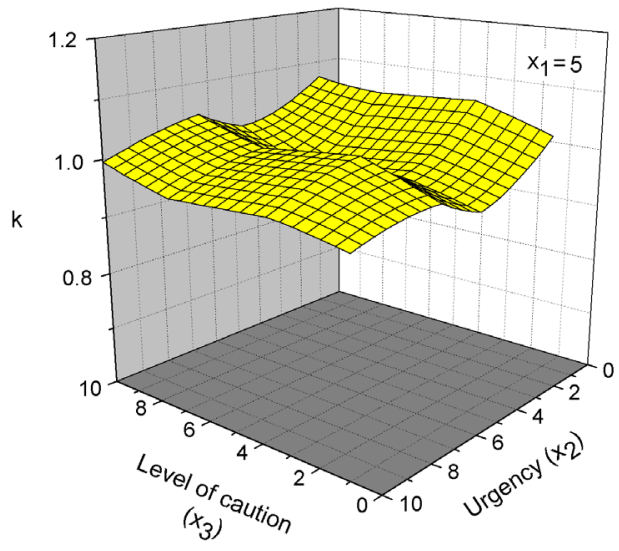

a) 


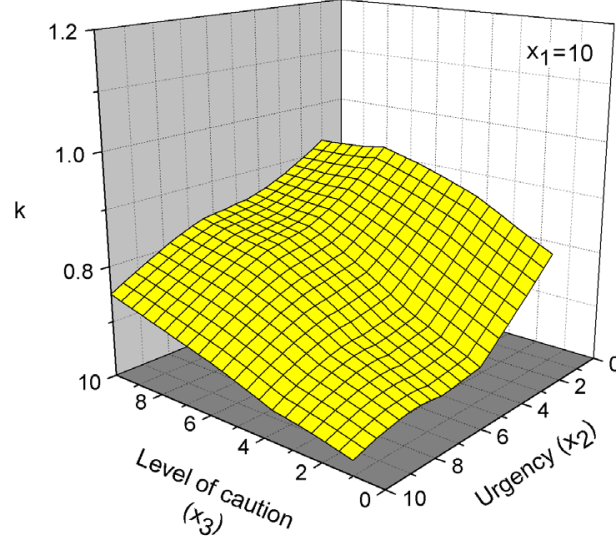

b)

Figure 4. Dependence of the correction factor on the urgency and the level of caution of transportation with satisfactory (a) and good (b) road conditions

Table 1. Correction factors of the model of transport-logistics infrastructure

\begin{tabular}{|c|c|c|c|}
\hline $\begin{array}{c}\text { Road } \\
\text { conditions }\end{array}$ & Urgency & $\begin{array}{c}\text { Level of } \\
\text { caution }\end{array}$ & $\begin{array}{c}\text { Correction } \\
\text { factor }\end{array}$ \\
\hline 1 & 4 & 9 & 1.42 \\
\hline 3 & 2 & 7 & 1.15 \\
\hline 5 & 9 & 3 & 0.98 \\
\hline 7 & 4 & 5 & 0.88 \\
\hline 8 & 8 & 10 & 0.89 \\
\hline 9 & & 6 & 0.77 \\
\hline
\end{tabular}

In the case of simulating a concrete transport network, the system of fuzzy inference of the Sugeno's type can be configured by selecting the parameters of linear dependency input-output.

\section{Conclusions}

Considered in the work method of formation of transport routes with the help of systems of fuzzy inference of the Sugeno's type allows obtaining quantitative values of factors that correct the weight of the edges of the graph in optimization problems under conditions of incomplete information and approximate input data. Such approach leads to an increase in the efficiency of transport-logistics infrastructure.

On the basis of computer simulation, it has been established that intelligent system based on fuzzy inference provides an increase in the weight of the edges of the graph in optimization algorithms for the formation of transport routes in the case of bad road conditions and high level of caution of transportation, and, conversely, reducing the weight of the ribs in the case of good road conditions or an increased urgency of transportation.

\section{REFERENCES}

[1] J. H. Bookbinder, T. A. Matuk. Logistics and Transportation in Global Supply Chains: Review, Critique and Prospects. Chapter 9 in M. Oskoorouchi (ed.). Tutorials in Operations Research, 182-211, 2009. DOI: 10.1287/educ. 1090.0059.

[2] R. L. Chapman, C. Soosay, J. Kandampully. Innovation in Logistic Services and the New Business Model: A Conceptual Framework, International Journal of Physical Distribution \& Logistics Management, Vol. 33, No. 7, 630 $650,2003$.

[3] S. J. Grawe. Logistics Innovation: A Literature-Based Conceptual Framework, International Journal of Logistics Management, Vol. 20, No. 3, 360-377, 2009.

[4] S. M. Wagner. Innovation Management in the German Transportation Industry, Journal of Business Logistics, Vol. 29, No. 2, 215-231, 2008.

[5] J. Żurek. E-commerce influence on changes in logistics processes, LogForum, Vol. 11, No. 2, 129-138, 2015.

[6] A. Perego, S. Perotti, R. Mangiaracina. ICT for Logistics and Freight Transportation: A Literature Review and Research Agenda, International Journal of Physical Distribution \& Logistics Management, Vol. 41, No. 5, 457-483, 2011.

[7] W. D. Kelton. Methodological Expectations for Studies Using Computer Simulation, Journal of Business Logistics, Vol. 37, No. 2, 82-86, 2016.

[8] A. M. Law. Simulation Modeling and Analysis (5 ed.), McGraw-Hill, New York, 2015.

[9] T. P. Stank, D. A. Pellathy, J. In, D. A. Mollenkopf, J. E. Bell. New Frontiers in Logistics Research: Theorizing at the Middle Range, Journal of Business Logistics, Vol. 38, No.1, 6-17, 2017.

[10] J. J. H. Liou, Y. T. Chuang. Developing a hybrid multi-criteria model for selection of outsourcing providers, Expert Systems with Applications, Vol. 37, No. 5, 3755-3761, 2010.

[11] T. H. Cormen, C. E. Leiserson, R. L. Rivest, C. Stein. Introduction to Algorithms (3rd.), MIT Press, 2009.

[12] L. A. Zadeh. Fuzzy Sets, Information and Control, Vol. 8, 338-353, 1965.

[13] K. Saravanan, N. M. Prabhu, B. R. Rajeswari. Fuzzy Controller Design of Lighting Control System by Using VI Package, International Journal of Scientific and Research Publications, Vol. 4, 1-6, 2014.

[14] V. Kumar, S. Kumar, H. Kansal. Fuzzy logic controller based operating room air condition control system, International Journal of Innovative Research in Electrical, Electronics, Instrumentation and Control Engineering, Vol. $2,510-514,2014$ 
[15] S. M. Sobhy, W. M. Khedr. Developing of Fuzzy Logic Controller for Air Condition System, International Journal of Computer Applications, Vol. 126, 1-8, 2015.

[16] T. Takagi, M. Sugeno. Fuzzy Identification of Systems and Its Applications to Modeling and Control, IEEE Transactions on Systems, Man and Cybernetics, Vol. 15, 116-132, 1985.
[17] T. Terano, K. Asai, M. Sugeno. Fuzzy Systems Theory and Its Applications, Academic Press, London, 1992.

[18] Y. Bai, D. Wang. Fundamentals of Fuzzy Logic Control Fuzzy Sets, Fuzzy Rules and Defuzzifications, Advanced Fuzzy Logic Technologies in Industrial Applications, Springer, 17-36, 2006. 